\title{
Immunoglobulin heavy variable (IgHV) gene mutation and micro-RNA expression in Burkitt's lymphoma at Moi Teaching and Referral Hospital in Western Kenya
}

\author{
Isaac Ndede ${ }^{1,2}$, SK Mining ${ }^{1}$, K Patel ${ }^{1}$, FM Wanjala ${ }^{2}$, C. Tenge ${ }^{3}$
}

1. Department of Immunology, Moi University School of Medicine, Eldoret, Kenya.

2. Department of Biological Sciences, University of Eldoret, Eldoret, Kenya.

3. Department of Child Health and Paediatrics, Moi University School of Medicine, Eldoret, Kenya.

\section{Emails:}

CT: cntenge@yahoo.co.uk; FMW: fmuyoma@gmail.com; SKM: smining57@gmail.com; KP: kirtikap@gmail.com; IN: iandede@yahoo.com;

\begin{abstract}
Introduction: Burkitt's lymphoma (BL) is a virus associated childhood B-cell cancer common in Eastern Africa. Continued survival of B-cells in germinal centres depend on expression of high affinity immunoglobulins (Ig) to complementary antigens by somatic hypermutation of Ig genes. Cellular microRNAs, non-coding RNAs have been reported to play role in cell cycle regulation. Both viral antigen dependent mutation and micro-RNA expression maybe involved in BL pathogenesis.

Objective: To describe immunoglobulin heavy variable ( $\mathrm{IgHV}$ ) rearrangement and micro-RNA expressions in BL tumours.

Methods: Genomic DNA were extracted and purified from BL tissue blocks at Moi Teaching and Referral Hospital, before amplification using IgHV consensus primers and sequencing. The sequences were then aligned with germline alleles in IMGT/ V-QUEST ${ }^{\circledR}$ database. Total RNA extracted from tissue blocks and cell lines were used to determine relative expression of hsamiR-34a and hsa-miR-127.

Results: In all tumours, allele alignment scores and number of mutations range were 89.2-93.2\%, 15-24 respectively. The range of IgHV amino acid changes were higher in EBER-1+ (15-25) than EBER-1- (9-15). In MYC+ tumours, the relative expression were: hsa-miR-127(2.09);hsa-miR-34a (2.8) and MYC- hsa-miR-127 (1.2), hsa-miR-34a (1.0).

Conclusion: B-cell in BL contained somatic mutated IgHV gene and upregulated cellular microRNAs with possible pathogenetic role(s).
\end{abstract}

Keywords: IgHV; somatic hypermutation; microRNA; Burkitt's lymphoma.

DOI: https://dx.doi.org/10.4314/ahs.v19i4.48

Cite as: Ndede I, Mining SK, Patel K, Wanjala FM, Tenge C. Immunoglobulin heavy variable (IgHV) gene mutation and micro-RNA expression in Burkitt's lymphoma at Moi Teaching and Referral Hospital in Western Kenya. Afri Health Sci.2019;19(4):3242-3248. bttps:/ / dx.doi.org/10.4314/ahs.v19i4.48

\section{Introduction}

Burkitt's lymphoma (BL) is common aggressive childhood non-Hodgkin lymphoma (NHL) endemic in sub-Saharan Africa (Orem, Katongole, Lambert, de Sanjose, \& Weiderpass, 2007). In this region, non-random

\section{Corresponding author: \\ Isaac Ndede, Department of Immunology, \\ Moi University School of Medicine, \\ P. O. Box 4606, Eldoret 30100, Kenya. \\ Tel. +254-723247938, \\ Email: iandede@yahoo.com}

and spatial-temporal clustering of BL appear to suggest relationship with prevalent infection with P. falciparum, Epstein Barr virus (EBV), Kaposi sarcoma-associated herpesvirus (KSHV), Human herpesvirus 8 (HHV-8) and Human immunodeficiency virus (HIV) (Rainey et al., 2006; Thapa, Li, Jamieson, \& Martínez-Maza, 2011). Persistent exposure to these pathogens thought to play a role in endemic BL pathogenesis (Mutalima et al., 2008). Normally B cells home in germinal centres (GC) where survival depend on their expression of high affinity immunoglobulin B-cell receptors (BCR) capable of recognising corresponding pathogen antigens. High-affinity receptor rearrangements are achieved through somatic hypermutation. A process in which germinal centre

(C) 2019 Ndede et al. Licensee African Health Sciences. This is an Open Access article distributed under the terms of the Creative commons Attribution License (https://creativecommons.org/licenses/BY/4.0), which permits unrestricted use, distribution, and reproduction in any medium, provided the original work is properly cited. 
B-cells acquire mutations at a high rate within the immunoglobulin complementarily-determining regions (CDR) including IgHV (Hecht \& Aster, 2000). Because somatic hypermutation is largely confined to germinal centre, its features therefore marks a B cell as being of GC or post GC origin. This apparent obvious GC derivation of many Burkitt's lymphoma contradicts the fact that some BL tumours growths are extra nodal and involve tissues that do not normally contain germinal centres under physiological condition (Cogliatti et al., 2006). The precise normal cell counterpart of B-cells in Burkitt's lymphoma is therefore unclear and controversial. The impact of infections such as EBV and posttranscription regulators such as microRNAs on B-cell differentiation has been intense discussion but remains largely unclear. It is known that lymphoid neoplasms in many respects recapitulate normal stages of lymphoid cell differentiation, activation, maturation and function (Jaffe, Harris, Stein, \& Isaacson, 2008), a fact useful for their classification and diagnosis.

MicroRNAs (miRNAs) are small endogenous non-coding RNAs approximately 21-23 nucleotides, long found in host and pathogens. Cellular miRNAs constitute approximately $1-3 \%$ of human genome of which about 940 have been identified (Sandhu, Croce, \& Garzon, 2011). MicroRNAs are critical gene regulators. They control about $30 \%$ of human genes by mediating cleavage and/or inhibition of genomic translation by binding to 3' untranslating region (UTR) of their target messenger RNAs (mRNA). Many miicroRNAs have been reported to influence cell growth, development and differentiation, making their dysregulation likely to result in malignancies including Burkitt's lymphoma (Esquela-Kerscher \& Slack, 2006). Due to their non-immunogenic property of microRNAs, viral miRNAs are used to execute viral latency to avoid immune response to viral antigens by the host (Lin \& Flemington, 2011).

Systematic profiling of microRNAs in lymphoma patient samples suggests a role in lymphomagenesis (Anna Onnis et al., 2010). Expression patterns of some microRNAs can provide insight into BL molecular phenotype and pathogenesis (Zhang et al., 2009), making them promising tools to explore the molecular signature of BL and other cancers (Lenze et al., 2011). Putting IgHV mutation and miRNA expression profiles together, may help to better describe and differentiate BL from other B cell lymphomas. We investigated B cell phenotype in selected BL tumours in terms of immunoglobueavy variable (IgHV) genes mutation and microRNA expression at Moi Teaching and Referral Hospital (MTRH) in western Kenya.

\section{Materials and methods}

A cross sectional study of children and adolescents aged $\leq 18$ years presenting with jaw or abdominal masses was carried out at Moi Teaching and Referral Hospital (MTRH) in western Kenya between 2012 and 2013. Initially, tissue sections were evaluated by histology and a panel of immunohistochemistry antibodies including EBER-1 and MYC. Demographic and clinical information were obtained from structured questionnaire and hospital files. Parental informed consent was obtained from each participant before recruitment. Patient information and specimen handling and processing were done in strict confidence. Institutional Research and Ethics Committee (IREC) of Moi University/MTRH approved the study, FAN 000654.

Genomic DNA extraction and sequencing was done on previously diagnosed BL cases using histology, immunohistochemistry and fluorescent in-situ hybridisation (FISH). The DNA was extracted from $20-\mu \mathrm{m}$-thick sections of formalin fixed paraffin embedded (FFPE) tissue blocks, by BioRobot EZ1 (Qiagen, Milan, Italy), following the manufacturer's protocol. Only extracts with DNA concentration $\geq 300 \mathrm{ng} / \mu \mathrm{l}$ were considered to contain sufficiently intact DNA for amplification and analysis for IGHV-IGHD-IGHJ rearrangement or somatic hypermutation. To avoid cross-contamination, all procedures performed before PCR amplification such as cutting of tissue sections and proteinase $\mathrm{K}$ digestion were performed in designated pre-amplification, amplification and post amplification sections of the laboratory. Polymerase chain reaction (PCR) amplifications were done on genomic DNA extracts using consensus primers for the IGHV framework region 1 (FR1), along with consensus J segment primers (BIOMED-2 Protocol) and multiplex identifier (MID) at each 5' end the primers to barcode the sample. Amplification efficiencies ranged from 50-100\%. The PCR amplification products were directly sequenced using ABI PRISM ${ }^{\circledR}$ BigDye Terminator v1.1 Ready Reaction Cycle Sequencing kit and ABI PRISM 310 Genetic Analyzer (both from Applied Biosystems, Germany). The resulting sequences were then analysed on ImMunoGene'Tics ${ }^{\circledR}$ (IMGT) database using IMGT/V-QUEST tool 
(version 3.3.5). This tool determines and localizes somatic mutations in immunoglobulin rearranged sequences by aligning with the database alleles.

\section{MicroRNA extraction and expression}

Total RNAs were extracted from about four (4) pieces of $5 \mu \mathrm{m}$ thick FFPE tissue sections each using miRNeasy® FFPE kit (Qiagen) following the manufacturer's instructions. Briefly, the tissue sections were deparaffinised, digested in proteinase $\mathrm{K}$, then heated on a Thermoblock at $80 \mathrm{oC}$ for 15 minutes followed by DNase treatment and binding to RNeasy MinElute $\mathbb{R}$ column, from where total RNA including microRNA were then eluted in a minimum quantity of $4 \mu \mathrm{l}$ RNase-free water. The amount and purity of RNA extracted were evaluated by measuring ratio of absorbance at $260 \mathrm{~nm}$ and $230 \mathrm{~nm}$ using Thermo Scientific Nanodrop ${ }^{\circledR}$ spectrophotometer (ND-1000, Nanodrop, Thermo Scientific $(\mathbb{R})$. A ratio of approximately 2.0 was considered as pure total RNA. A lower ratio indicates protein contaminants. Expression of cellular miRNA levels were then evaluated using real-time quantitative reverse transcription (qRT-PCR) using TaqMan ${ }^{\circledR}$ MicroRNA Assay kit according to the manufacturer's instructions. Specific cDNA were prepared using specific TaqMan ${ }^{\circledR}$ primers for hsa-miR-34a, hsa-miR-127 and
RNU-43 (\#4366596 Applied Biosystems, Germany) by means of reverse transcription kit. The expression levels of miRNAs were normalized to that of RNU43, which was stably expressed among the samples. Controls were prepared from paraffin-embedded blocks made from Cag (EBV-positive) and Ramos (EBV-negative) cell lines. The data generated were analysed using proprietary software DataAssist ${ }^{\circledR}$ to derive relative quantity of miRNA expression.

\section{Data management}

The study data were entered into computer for storage and initial analysis. Descriptive analyses were performed by using frequency tables, for categorical variables using $\mathrm{R}$ Studio version 1.1. For continuous variables such as age, means and other relevant measures of central tendency were used.

\section{Results}

There were a total of thirty three children and adolescents with Blin this series and consisted of 78.8\%,21.8\% males and females respectively. Of the randomly selected four BL cases, there were 3 males and 1 female aged between 4 and 12 years, tumour site; 3 jaw, 1 abdominal with variable EBER-1 and MYC status (Table 1).

Table 1. Case gender, age tumour site and EBER-1 status

\begin{tabular}{lllll}
\hline Case & Sex & Age, $\mathbf{y}$ & Tumour site & EBER-1 \\
\hline $\mathbf{1}$ & female & 4 & Jaw & Positive \\
$\mathbf{2}$ & Male & 7 & Abdominal & Positive \\
$\mathbf{3}$ & Male & 6 & Jaw & Positive \\
$\mathbf{4}$ & Male & 12 & jaw & Negative \\
\hline
\end{tabular}

Cases 1-3 were positive for EBER-1 while case 4 was negative for EBER-1 (Table 2). After aligning DNA amplicons sequences with the closest $\mathrm{V}$-regions alleles from the IMGT/V-QUEST reference database, percentage nucleotide identity were: case1 $(89.2 \%)$ case $2(93.2 \%)$, case3 $(90.4 \%)$, case4 (89.2\%) (Figures 1 \& Table 2). 


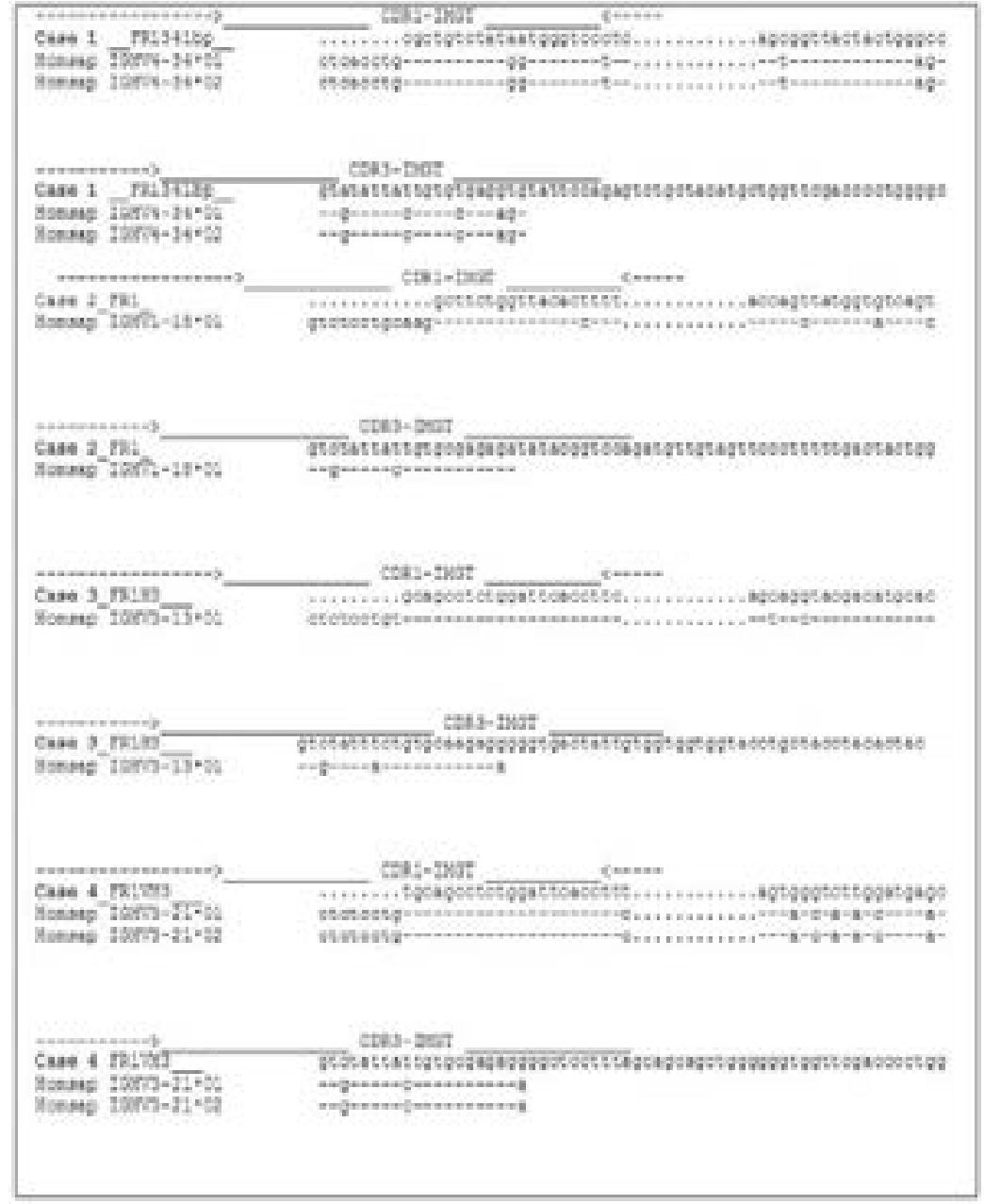

Figure 1. CDR alignment with corresponding alleles. Dashes indicate identical nucleotides. Dots indicate gaps or nucleotides not taken into account for the alignments. Nucleotide identity $(\%)$ cases $1=90.5$, case $2=93.2$, case $3=90.4$, case $4=89.2$.

Table 2. IgHV gene and closest germline allele's usage (\%), $\mathrm{V}$ region mutations

\begin{tabular}{lcccccc}
\hline Case & Allele & $\begin{array}{c}\text { V-gene \& allele } \\
\text { Identity (\%) }\end{array}$ & $\begin{array}{l}\text { V-region } \\
\text { mutations }\end{array}$ & $\begin{array}{l}\text { Amino } \\
\text { acid changes }\end{array}$ & $\begin{array}{l}\text { CDR-IMGT } \\
\text { lengths }\end{array}$ & EBER+/- \\
\hline $\mathbf{1}$ & HomsapIgHV4-34*01 F & 90.5 & 22 & 14 & 8.7 .14 & Positive \\
$\mathbf{2}$ & HomsapIgHV1-18*01 F & 93.2 & 15 & 9 & 8.8 .15 & Positive \\
$\mathbf{3}$ & HomsapIgHV-13*01 F & 90.4 & 22 & 13 & 8.7 .20 & Positive \\
$\mathbf{4}$ & HomsapIgHV3-21*01 F & 89.2 & 25 & 14 & 8.8 .15 & Negative \\
\hline
\end{tabular}


The number of mutation (amino acid changes) in the V-region fromFR1 toFR3 compared to the closest germline V-genes of similar IMGT/CDR lengths were: case1; 22(14), case2; 15(9), case3; 22(13) and case4; 25(14) regardless of EBER-1 status (Table 2). Relative expression

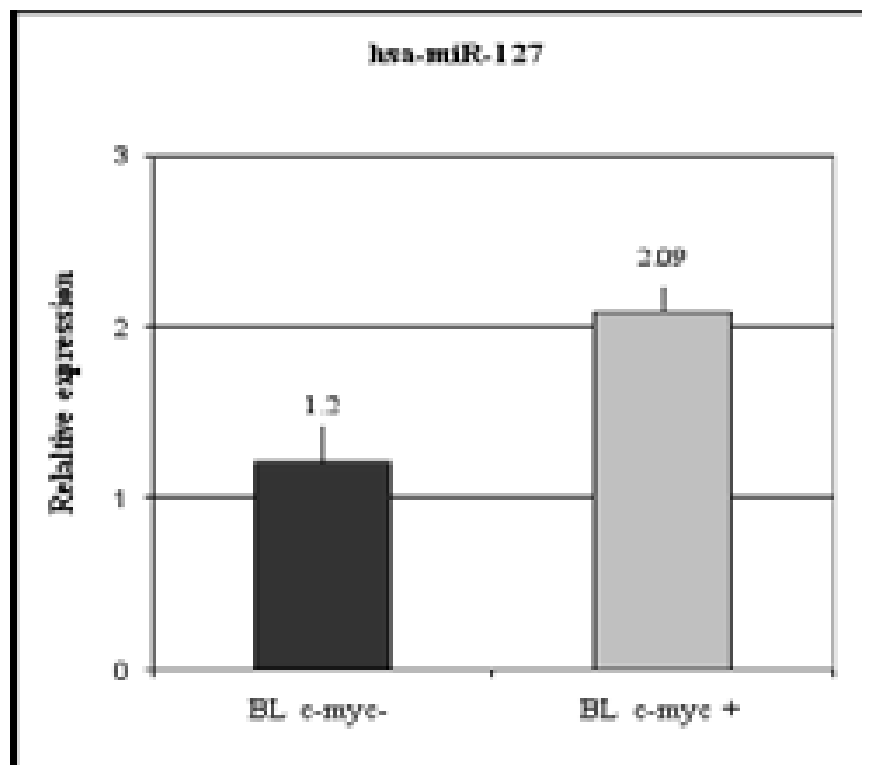

a)

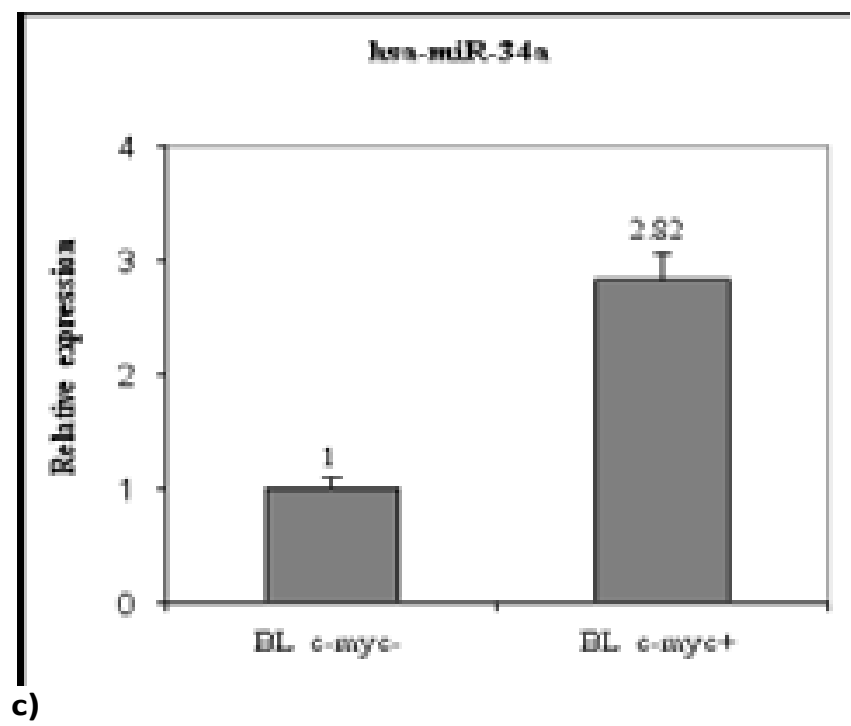

of cellular microRNAs, hsa-miR-127 and hsa-miR-34a were higher (2.8 vs 2.09) in MYC-positive BL compared to MYC-negative BL cases, Ramos (EBER-1-negative) and Cag (EBER-1 positive), a multiple myeloma cell lines (Figure 2).

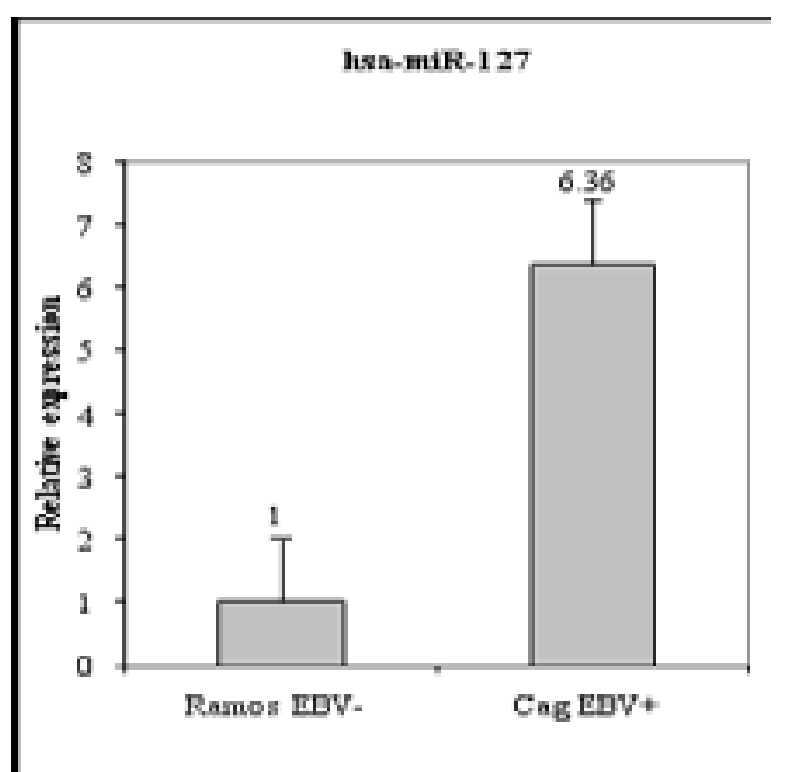

b)

Figure 2. Relative expression of; a) $h s a-m i R-127$ in BL cases, b) $h s a-m i R-127$, in Ramos and Cag cell lines and c) hsa-miR-34a

\section{Discussion}

In this study, IgHV DNA input sequences of Burkitt's lymphoma BCRs harbour mutations compared to their corresponding germline alleles in IMGT/V QUEST database suggesting presence of somatic mutations possibly due to interactions with an extrinsic pathogen antigen in the germinal centres. This may imply that these B cells in the studied tumours are antigen experienced and therefore supports GC phenotype of BL tumours cells as previously reported (God \& Haque, 2010). Amato et al (2016) also reported a higher mutation frequency in EBV associated BL compared to sporadic BL, supporting view that antigen selection pressure may be playing a role in the pathogenetic pathway of endemic BL. In 
this current study, both EBER-1 positive and negative tumours showed evidence of $\mathrm{V}$-gene mutation in $\mathrm{IgHV}$ BCR. The fact that EBV encoded products detected in these tumours is consistent with Navari et al (2018) that Epstein-Bar virus encoded RNA may contribute pathogentic effects by interfering with diverse cellular mechanisms including immunosurveillance, cell proliferation and apoptosis. On the other hand, some studies have reported BL with undetected EBV encoded products (Leucci et al.,2010). The absence of EBER-1 in some but not all BL tumours may mean that in some BL, the malignant B cells have different maturation stage(s) compared to their EBER-1 positive counterparts. It is noteworthy that all the BL tumours in this study stained positive staining for CD20 and CD10 (results not shown), which are known are markers associated with centroblast and germinal centre (GC) B cells respectively. But this apparent GC derivation contrasts a frequent observation that some BL tumour growths are extra nodal and do not involve tissues that contain germinal centres under physiological conditions. Thus the IgHV mutations observed in these tumours, in addition to CD10 and CD20 phenotype appear to suggest that B cells in the studied tumours are most likely derived from centroblast, germinal centre or post-germinal centre and are antigen experienced. This supports findings by Tamaru et al (1995) that BL cells are characterised by antigen driven somatic hypermutated $\mathrm{IgVH}$ genes. Thus, neoplastic B cells in BL appear to arise either from early centroblasts or GC stages that are arrested after somatic hypermutation. Germinal centre phenotype and/or MYC expression in some BL tumours, suggest probable errors in class-switch recombination stage Piccaluga et al (2011). The differences in cells of origin of BL tumours supports the view that there are different pathologic mechanisms or entities of BL (Bellan et al., 2003)

This study reports, increased relative expressions of hsamiR-34 and hsa-miR-127 in c-myc and EBV positive BL compared to Ramos cell line, an EBV-negative BL derived cell lineThis evidence supports of those by Leucci et al (2008) that viral encoded products c myc expression and host miroRNA pattern.. Studies by Onnis et al (2012) also reported that EBV encorded nuclear antigen-1 induce host hsa miR- 127 which acts post transcriptionally to dysregulate memory B cells, leading to BL pathogene- sis . The high expression of hsa-miR-34a by c myc positive cell lines further suggests a networking relationship between pathogen antigen, viral and host microRNAs. Blockade of $\mathrm{B}$ cell differentiation by increased hsa miR34a expression has been proposed (Lisio et al., 2012). The role of EBV and/or microRNAs lymphoma pathogenesis is evident.

\section{Conclusion}

B cells in Burkitt's lymphoma tumour studied here contained rearranged Immunoglobulin heavy variable ( $\mathrm{IgHV}$ ) region genes, antigen driven somatic hypermutations with of CD10 and CD20 phenotype, suggesting either GC or post GC derivation. Higher relative expressions of cellular microRNAs hsa-miR 34a and hsa-miR 127 in EBER-1 and MYC+ positive BL tumours further implicate miRNAs and pathogen antigens in lymphomagenesis, and can provide potential therapeutic applications. Profiling other microRNAs and mutation in larger BL series is recommended.

\section{Conflict of interest}

None declared.

\section{References}

Amato, T., Abate, F., Piccaluga, P., Iacono, M., Fallerini, C., Renieri, A., ... Bellan, C. (2016). Clonality Analysis of Immunoglobulin Gene Rearrangement by Next-Generation Sequencing in Endemic Burkitt Lymphoma Suggests Antigen Drive Activation of BCR as Opposed to Sporadic Burkitt Lymphoma. American Journal of Clinical Pathology, 145, 116-127.

Bellan, C., Lazzi, S., Falco, G. De, Nyongo, A., Giordano, A., \& Leoncini, L. (2003). Burkitt's lymphoma: new insights into molecular pathogenesis. Journal of Clinical Pathology, 56, 188-192.

Cogliatti, S. B., Novak, U., Henz, S., Schmid, U., Moller, P., \& Barth, T. F. E. (2006). Diagnosis of Burkitt lymphoma in due time: a practical approach. British Journal of Haematology, 134(3), 294-301.

Esquela-Kerscher, A., \& Slack, F. J. (2006). MicroRNAs with a role in cancer. Nature Reviews. Cancer, 6(4), 256269.

God, J. M., \& Haque, A. (2010). Burkitt Lymphoma: Pathogenesis and Immune Evasion. Journal of Oncology, 2003-2005. 
Hecht, J. L., \& Aster, J. C. (2000). Molecular Biology of Burkitt' s Lymphoma. Journal of Clinical Oncology, 18(21), 3707-3721.

Jaffe, E. S., Harris, N. L., Stein, H., \& Isaacson, P. G. (2008). Classification of lymphoid neoplasms: the microscope as a tool for disease discovery. Blood, 112(12), 4384-4399.

Lenze, D., Leoncini, L., Hummel, M., Volinia, S., Liu, C. G., Amato, T., ... Stein, H. (2011). The different epidemiologic subtypes of Burkitt lymphoma share a homogenous micro RNA profile distinct from diffuse large B-cell lymphoma. Leukemia, 25(12), 1869-1876.

Leucci, E., Cocco, M., Onnis, A., Falco, G. De, Cleef, P. Van, Bellan, C., ... Leoncini, L. (2008). MYC translocation-negative classical Burkitt lymphoma cases : an alternative pathogenetic mechanism involving miRNA deregulation, (July), 440-450.

Leucci, E., Onnis, A., Cocco, M., De Falco, G., Imperatore, F., Giuseppina, A., ... Leoncini, L. (2010). B-cell differentiation in EBV-positive Burkitt lymphoma is impaired at posttranscriptional level by miRNA-altered expression. International Journal of Cancer, 126(6), 1316-1326. Lin, Z., \& Flemington, E. K. (2011). miRNAs in the pathogenesis of oncogenenic human viruses. Cancer Letters, 305(2), 186-199.

Lisio, L. Di, Martinez, N., Montes-moreno, S., Piris-villaespesa, M., Sanchez-beato, M., \& Paris, M. A. (2012). The role of miRNAs in the pathogenesis and diagnosis of B-cell lymphomas Review article The role of miRNAs in the pathogenesis and diagnosis of B-cell lymphomas. Blood, 120(9), 1782-1790.

Mutalima, N., Molyneux, E., Jaffe, H., Kamiza, S., Borgstein, E., Mkandawire, N., ... Newton, R. (2008). Associations between Burkitt Lymphoma among Children in Malawi and Infection with HIV, EBV and Malaria: Results from a Case-Control Study. PLOS ONE, 3(6), e2505 . Navari, M., Etebari, M., Ibrahimi, M., Leoncini, L., \& Piccaluga, P. P. (2018). Pathobiologic Roles of Epstein - Barr Virus-Encoded MicroRNAs in Human Lymphomas. International Journal of Molecular Scieences, 19, 1168.
Onnis, A., Falco, G. De, Antonicelli, G., Onorati, M., Bellan, C., Sayed, S., \& Leoncini, L. (2010). Alteration of MicroRNAs Regulated by c-Myc in Burkitt Lymphoma, 5(9).

Onnis, A., Navari, M., Antonicelli, G., Morettini, F., Mannucci, S., De Falco, G., ... Leoncini, L. (2012). Epstein-Barr nuclear antigen 1 induces expression of the cellular microRNA hsa-miR-127 and impairing B-cell differentiation in EBV-infected memory B cells. New insights into the pathogenesis of Burkitt lymphoma. Blood Cancer Journal, 2(8), e84.

Orem, J., Katongole, E., Lambert, B., de Sanjose, S., \& Weiderpass, E. (2007). Burkitt's lymphoma in Africa , a review of the epidemiology and etiology. African Health Sciences, 7(3), 166-175.

Piccaluga, P. P., Falco, G. De, Kustagi, M., Gazzola, A., Agostinelli, C., Tripodo, C., ... Leoncini, L. (2011). Gene expression analysis uncovers similarity and differences among Burkitt lymphoma subtypes subtypes. Blood, 117, 3596-3608.

Rainey, J. J., Omenah, D., Sumba, P. O., Moormann, A. M., Rochford, R., \& Wilson, M. L. (2006). Spatial clustering of endemic Burkitt's lymphoma in high-risk regions of Kenya. International Journal of Cancer, 127, 121-127.

Sandhu, S. K., Croce, C. M., \& Garzon, R. (2011). Micro-RNA Expression and Function in Lymphomas. Advances in Hematology, 2011, 347137.

Tamaru, J., Hummel, M., Marafioti, T., Kalvelage, B., Leoncini, L., Minacci, C., ... Stein, H. (1995). Burkitt's Lymphomas Express VH Genes with a Moderate Number of Antigen-Selected Somatic Mutations, 147(5), 1398-1407.

Thapa, D. R., Li, X., Jamieson, B. D., \& Martínez-Maza, O. (2011). Overexpression of microRNAs from the miR17-92 paralog clusters in AIDS-related non-Hodgkin's lymphomas. PloS One, 6(6), e20781.

Zhang, J., Jima, D. D., Jacobs, C., Fischer, R., Gottwein, E., Huang, G., ... Dave, S. S. (2009). Patterns of microRNA expression characterize stages of human B-cell differentiation. Blood, 113(19), 4586-4594. 